\title{
Human Galectins Induce Conversion of Dermal Fibroblasts into Myofibroblasts and Production of Extracellular Matrix: Potential Application in Tissue Engineering and Wound Repair
}

\author{
Barbora Dvořánkováa, d Pavol Szabo ${ }^{a, d} \quad$ Lukas Lacina $^{a, b, d}$ Peter Gal ${ }^{a, f, g}$ \\ Jana Uhrovac Tomas Zimac Herbert Kaltner ${ }^{\mathrm{h}}$ Sabine Andréh \\ Hans-Joachim Gabius ${ }^{\text {h }}$ Eva Sykovad, e Karel Smetana, Jr. a, d \\ a Institute of Anatomy, ${ }^{b}$ Department of Dermatovenerology, ${ }^{\mathrm{C}}$ Institute of Clinical Biochemistry and Laboratory \\ Diagnostics, 1st Faculty of Medicine, dCenter of Cell Therapy and Tissue Repair, 2nd Faculty of Medicine, Charles \\ University, and ${ }^{\mathrm{e}}$ Institute of Experimental Medicine, Academy of Sciences of the Czech Republic, Prague, Czech \\ Republic; f Department for Biomedical Research, East-Slovak Institute of Cardiovascular Diseases, ${ }^{9}$ Department \\ of Pathological Anatomy, University of Veterinary Medicine and Pharmacy, Košice, Slovak Republic; ${ }^{\mathrm{h}}$ Institute of \\ Physiological Chemistry, Faculty of Veterinary Medicine, Ludwig-Maximilians-University Munich, Munich, Germany
}

\section{Key Words}

Extracellular matrix $\cdot$ Fibronectin $\cdot$ Keratinocyte $\cdot$ Lectin $\cdot$ Tissue engineering

\footnotetext{
Abstract

Members of the galectin family of endogenous lectins are potent adhesion/growth-regulatory effectors. Their multifunctionality opens possibilities for their use in bioapplications. We studied whether human galectins induce the conversion of human dermal fibroblasts into myofibroblasts (MFBs) and the production of a bioactive extracellular matrix scaffold is suitable for cell culture. Testing a panel of galectins of all three subgroups, including natural and engineered variants, we detected activity for the proto-type galectin-1 and galectin-7, the chimera-type galectin-3 and the tandemrepeat-type galectin-4. The activity of galectin-1 required the integrity of the carbohydrate recognition domain. It was independent of the presence of TGF- $\beta 1$, but it yielded an ad-
}

ditive effect. The resulting MFBs, relevant, for example, for tumor progression, generated a matrix scaffold rich in fibronectin and galectin-1 that supported keratinocyte culture without feeder cells. Of note, keratinocytes cultured on this substratum presented a stem-like cell phenotype with small size and keratin-19 expression. In vivo in rats, galectin-1 had a positive effect on skin wound closure 21 days after surgery. In conclusion, we describe the differential potential of certain human galectins to induce the conversion of dermal fibroblasts into MFBs and the generation of a bioactive cell culture substratum.

Copyright $\odot 2011$ S. Karger AG, Basel

\section{Abbreviations used in this paper}

ECM extracellular matrix

MFBs myofibroblasts

\section{KARGER}

Fax +4161306 1234

E-Mail karger@karger.ch

www.karger.com
(C) 2011 S. Karger AG, Basel

$1422-6405 / 11 / 1946-0469 \$ 38.00 / 0$

Accessible online at:

www.karger.com/cto
Dr. Karel Smetana

Institute of Anatomy, 1st Faculty of Medicine, Charles University

U Nemocnice 3

CZ-128 00 Prague 2 (Czech Republic)

Tel. +420 22496 5873, E-Mail karel.smetana@lf1.cuni.cz 


\section{Introduction}

Stem cells hold an enormous therapeutic potential, but obstacles remain to be overcome for their successful clinical application, for example, concerning the generation of a suitable microenvironment to maintain stemness and to ensure anchorage [Voog and Jones, 2010]. In this context, the components of the extracellular matrix (ECM) play pivotal roles in docking cells and engaging them in the complex molecular interplay essential for the cells' functionality. As a consequence, aberrations in this complex network, either engineered in mice or detected in patients, can lead to diseases [Hennet, 2009; Honke and Taniguchi, 2009]. Such observations guide interest especially to the glycan part of the ECM, a highly versatile platform for coding biochemical information, as embodied in the concept of the sugar code [Gabius, 2009]. Indeed, simple monosaccharides used as a matrix, inspired by this bioactivity of glycans, can already serve as a bioactive substratum, even substituting for feeder cells in the case of epidermal keratinocytes [Labský et al., 2003]. In order to achieve the natural complexity, it would be ideal to direct cells to produce suitable ECM using endogenous effectors.

Toward this end, observations that an endogenous lectin, such as galectin-1, has proven capable of promoting the attachment of diverse types of cells, also in concert with matrix glycoproteins or chitosan, have given our research a clear direction [André et al., 1999; Chang et al., 2004; Kadri et al., 2005]. This type of galectin binds the $\alpha_{5} \beta_{1}$-integrin, as well as the associated ganglioside GM1, that influences cell adhesion and growth [André et al., 2007; Wang et al., 2009]. It can redirect gene expression, for example of $\alpha 2$ (I) collagen via TGF- $\beta / S m a d 3$ signaling, and the exposure of fetal skin or mesenchymal stem cells leads to conversion to the myogenic lineage and even mature myotubes in vitro [Goldring et al., 2002a, b; Fischer et al., 2005; Chan et al., 2006; Okano et al., 2008]. In addition to potential medical relevance, we have detected a strong galectin-1 presence in the stroma of squamous cell carcinomas and in healing wounds [Lacina et al., 2007; Klíma et al., 2009]. Here, the presence of myofibroblasts (MFBs), a distinct type of fibroblast positive for $\alpha$-smooth muscle actin, is frequent [Hinz 2007; De Wever et al., 2008]. Their generation by conversion in situ is assumed to be induced by TGF- $\beta$ [Brenmoehl et al., 2009], their presence then contributing to promote wound healing/contraction and tumor progression of squamous carcinoma cells. Obviously, the ECM of MFBs can be conducive in programming cell activities. This concept led us to test the hypothesis whether galectin-1 is involved in MFB and ECM generation and then to investigate its bioactivity as a culture substratum for human keratinocytes. In other words, galectin-1 was studied as a potential inducer of the conversion of adult dermal fibroblasts into MFBs, using TGF- $\beta 1$ and TGF- $\beta 3$ as positive controls. Following these experiments, we addressed the issue of measuring the profile of this activity within the family of human galectins. This family consists of three groups, which differ in the spatial presentation of the lectin domain, with characteristic expression in human skin [Villalobo et al., 2006; Čada et al., 2009]. As a consequence, homodimeric galectin-1, galectin-2 and galectin-7, the chimeric galectin-3 and the tandem-repeat-type galectin- 4 and galectin- 8 were comparatively studied under identical conditions. Natural variants, that is, truncated galectin-3 after proteolytic removal of the stalk region and the two galectin-8 forms differing in linker length, as well as an engineered form of galectin- 4 without linker and a galectin-1 mutant without lectin activity, completed the test panel used in the present study. In addition to testing the ability of the lectins in the panel to induce MFBs and the capacity of their ECM to support keratinocyte culture and for wound healing in vivo, we also contribute to defining the extent of functional divergence among these homologous endogenous lectins.

\section{Materials and Methods}

\section{Preparation of Galectins}

Human galectins (table 1), including the linkerless variant of galectin- 4 and the E71Q mutant of galectin-1, were obtained by recombinant production and purified by affinity chromatography on lactosylated Sepharose $4 \mathrm{~B}$ (or Ni-CAM ${ }^{\mathrm{TM}} \mathrm{HC}$ resin from Sigma, Munich, Germany, in the case of the His-tagged galectin-1 mutant without lectin activity) as a crucial step [Gabius et al., 1985], routinely followed by the removal of any lipopolysaccharide contamination [Sarter et al., 2009]. Product analysis was performed using one- and two-dimensional gel electrophoresis, gel filtration and mass spectrometry as well as hemagglutination, solid-phase and cell assays to ascertain activity [André et al., 2006, 2007, 2008; Beer et al., 2008]. Proteolytic truncation of galectin-3 with cleavage at the Tyr106/Gly107 and Glu229/Ile230 peptide bonds was performed using collagenase digestion [Kopitz et al., 2001; Kübler et al., 2008].

\section{The Influence of Galectins on Human Dermal Fibroblasts}

Dermal fibroblasts were isolated from skin specimens obtained from healthy donors with their informed consent (in agreement with the Helsinki Declaration after approval by the Local Ethical Committee) in the Department of Aesthetic Surgery (Charles University, 3rd Faculty of Medicine). Sterile solutions of human galectins were prepared in Dulbecco's culture medium 
Table 1. Galectins used in experiments

\begin{tabular}{lll}
\hline Type of galectin & Galectin & Comment \\
\hline $\begin{array}{ll}\text { Homodimeric } \\
\text { proto-type) }\end{array}$ & $\begin{array}{ll}\text { galectin-1 } \\
\text { galectin-1-E71Q }\end{array}$ & strongly reduced affinity for carbohydrates \\
\cline { 2 - 3 } galectin-2 & \\
\cline { 2 - 3 } galectin-7 & galectin-3 & $\begin{array}{l}\text { proteolytically processed form lacks the collagenase-sensitive N-terminal section, reduc- } \\
\text { ing ability for cross-linking }\end{array}$ \\
\cline { 2 - 3 } Tandem-repeat & galectin-3-truncated & $\begin{array}{l}\text { prototype-like galectin-4 with covalent domain linkage after engineered removal of the } \\
\text { linker peptide }\end{array}$ \\
\cline { 2 - 3 } & galectin-8-short & $\begin{array}{l}\text { different lengths of the linker } \\
\text { galectin-8-long }\end{array}$ \\
\hline
\end{tabular}

(DMEM) containing 10\% fetal calf serum and antibiotics (all from Biochrom, Berlin, Germany). Human dermal fibroblasts were seeded at a density of 2,000 cells $/ \mathrm{cm}^{2}$ and cultured for 3-4 weeks. The culture medium was changed three times per week. The sensitivity of fibroblasts to TGF- $\beta 1 /-\beta 3$ was tested at $10 \mathrm{ng} /$ $\mathrm{ml}$, as described elsewhere [Brenmoehl et al., 2009]. The contribution of galectins to MFB formation was assessed by using galectins (table 1) in concentrations of 200, 300, 400 and $600 \mathrm{ng} / \mathrm{ml}$. The additive effect of both galectin- 1 and TGF- $\beta 1(10 \mu \mathrm{g} / \mathrm{ml})$ was tested by incubating cells with both substances. The role of galectin was, moreover, verified by application of $20 \mu \mathrm{g} / \mathrm{ml}$ of a polyclonal anti-galectin-1 antibody, developed in our laboratory and carefully tested for lack of cross-reactivity with other types of galectins (please see below). To exclude the nonspecific effect of immunoglobulins, the commercial anti-thyroglobulin rabbit polyclonal antibody (Dako, Glostrup, Denmark) was also tested.

Using a commercial ELISA kit (R\&D Systems, Minneapolis, Minn., USA), the concentration of TGF- $\beta 1$, the best-known inducer of MFB formation, was measured in conditioned media from cultures of human dermal fibroblasts in the absence or presence of galectins in a Multiskan RC reader (Labsystem, Helsinki, Finland) at $450 \mathrm{~nm}$.

\section{Human Keratinocytes and Their Culture on Matrices}

Induced by Galectins

The ECM scaffolds produced by cells exposed to galectins on the surface of coverslips were tested as substrates for the culture of human keratinocytes obtained as described above. The coverslips with the three-dimensional scaffolds were incubated with sterile distilled water for $60 \mathrm{~min}$ to devitalize MFBs by osmotic shock (as ascertained by subsequent culture), then the supports were incubated with culture medium for $24 \mathrm{~h}$ to prevent osmotic stress during the subsequent culturing of keratinocytes. These cells were obtained from the same clinical source and under identical ethical conditions as the fibroblasts. They were seeded at a density of $40 \times 10^{3}$ cells $/ \mathrm{cm}^{2}$ and cultured in keratinocyte medium [Strnad et al., 2009] at $5 \% \mathrm{CO}_{2}$ and $37^{\circ} \mathrm{C}$ for 1 week. Part of these experimental acellular supports with three-dimensional ECM lattices was fixed with $0.25 \%$ glutaraldehyde (Sigma-Ald- rich, Prague, Czech Republic) for $30 \mathrm{~min}$ at laboratory temperature. After extensive washing they were used as described above. Cells cultured on untreated coverslips were used as a control.

\section{Immunocytochemical Analysis of Cultured Cells}

The staining procedure, including fluorescence profiling, had been described recently in detail [Szabo et al., 2009]. Briefly, the tested specimens were fixed with $2 \%$ buffered paraformaldehyde ( $\mathrm{pH}$ 7.2-7.4) for $5 \mathrm{~min}$ and washed with PBS. The cells were permeabilized by exposure to Triton X-100 (Sigma-Aldrich), and sites for the antigen-independent adsorption of antibodies were blocked by bovine serum albumin and/or nonimmune porcine serum, serum application preventing the binding of antibodies via the interaction of the Fc fragments of immunoglobulins with cellular Fc receptors. Commercial antibodies were diluted as recommended by the suppliers. The primary and secondary antibodies used in this study are summarized in table 2 . The lack of crossreactivity of our homemade anti-galectin antibodies was ascertained, if required, after affinity depletion on resin with covalenty conjugated galectins [Kaltner et al., 2002; Saal et al., 2005]. The specificity of the immunohistochemical reaction was verified by the replacement of specific antibodies by an irrelevant antibody recognizing thyroglobulin instead of the first-step antibody and/or by the omission of the first-step antibody as well as by processing positive control specimens. Cell nuclei were counterstained by 4',6-diamidino-2-phenylindole (DAPI; Sigma-Aldrich). The specimens were mounted in Vectashield (Vector Laboratories, Burlingame, Calif., USA) and inspected by an Eclipse 90i fluorescence microscope (Nikon, Prague, Czech Republic) equipped with filter blocks for FITC, TRITC and DAPI, and a Cool-1300Q CCD camera (Vosskühler, Osnabrück, Germany); data were analyzed by a LUCIA 5.1 computer-assisted image analysis system (Laboratory Imaging, Prague, Czech Republic).

\section{Experimental Animals and in vivo Study}

The study design with 1-year-old male Sprague-Dawley rats $(\mathrm{n}=48)$ was approved by the Ethical Committee of the Faculty of Medicine of Šafárik University and by the State Veterinary Administration of the Slovak Republic. Surgery was performed un- 
Table 2. Primary and secondary antibodies

\begin{tabular}{lll}
\hline Antigen & Type of antibody & Source \\
\hline Gal-1, Gal-2, Gal-3, Gal-4, Gal-7 & rabbit polyclonal & $\begin{array}{l}\text { Faculty of Veterinary Medicine, Ludwig-Maximilians-University, } \\
\text { Munich, Germany }\end{array}$ \\
\hline Gal-1 & mouse monoclonal & Vector Laboratories, Burlingame, Calif., USA \\
\hline Fibronectin & rabbit polyclonal & Dako \\
\hline Fibronectin & mouse monoclonal & Abcam, Cambridge, UK \\
\hline $\begin{array}{l}\text { Collagen-1 } \\
\text { Collagen-3 }\end{array}$ & $\begin{array}{l}\text { mouse monoclonal } \\
\text { mouse monoclonal }\end{array}$ & Sigma-Aldrich, Prague, Czech Republic \\
\hline Smooth muscle actin & mouse monoclonal & Dako \\
\hline Keratin (wide spectrum) & rabbit polyclonal & Abcam \\
\hline $\begin{array}{l}\text { Keratin-19 } \\
\text { Thyroglobulin }\end{array}$ & $\begin{array}{l}\text { mouse monoclonal } \\
\text { mouse monoclonal } \\
\text { rabbit polyclonal }\end{array}$ & Dako \\
\hline $\begin{array}{l}\text { Rabbit immunoglobulins } \\
\text { Mouse immunoglobulins }\end{array}$ & $\begin{array}{l}\text { FITC-labeled swine serum } \\
\text { FITC-labeled swine serum }\end{array}$ & Alseva, Prague, Czech Republic \\
\hline $\begin{array}{l}\text { Mouse immunoglobulins } \\
\text { TRITC-labeled goat serum }\end{array}$ & Sigma-Aldrich \\
\hline
\end{tabular}

der general anesthesia induced by the administration of ketamine (40 mg/kg; Narkamon a.u.v.; Spofa, Prague, Czech Republic), xylazine (15 mg/kg; Rometar a.u.v.; Spofa) and tramadol (5 mg/kg; Tramadol-K; Krka, Novo Mesto, Slovenia). One full-thickness incision, $4 \mathrm{~cm}$ long, was made on the back of each rat. Cranially and caudally from the incision, round full-thickness skin excisions, $1 \mathrm{~cm}$ in diameter, were performed. The incision was then closed using an intradermal running suture (Chiraflon 5/0; Chirmax, Prague, Czech Republic). Subsequently, the rats were randomly assigned to four groups: (1) testing wild-type galectin-1, (2) testing the E71Q mutant, (3) testing full-length galectin-3 and (4) mock treatment. Wounds of rats from groups 1-3 were treated daily with the respective galectin starting from the day of surgery until the third postoperative day. All rats, including the control group, were kept in cages with an oval opening over the wound to ensure similar levels of stress for each animal during the experiment.

The healing of wounds was measured from standardized photographs as follows. Wounds were photographed with a scale immediately after surgery and at days 7 and 21 using an Olympus E330 digital camera equipped with a digital ED $50 \mathrm{~mm}$ f $2.0 \mathrm{mac}-$ ro objective and a ring set flash SRF-11 (Olympus, Tokyo, Japan). The wound area was then measured on the images using Quick Photo Micro 2.2 software (Premiere, Prague, Czech Republic) and expressed as a percentage of the original wound area generated on the day of surgery to reflect the level of wound healing. Statistical processing of the measured data was done using one-way ANOVA followed by the Tuckey-Kramer post hoc test. The quality of wound healing was evaluated in paraffin-embedded sections stained with hematoxylin-eosin.

\section{Results}

\section{In vitro Experiments}

Galectins Induce MFB Formation

Cultures of normal dermal fibroblasts contained none or only a very limited number of MFBs (fig. 1a, b). To test the effector activity of the galectins, we systematically used galectin- 1 and detected a significant increase in the number of MFBs in the third week of culture (fig. 1c). Next, we tested all the selected galectins (galectin-1, galectin-1-E71Q mutant, galectin-2, galectin-3, truncated version of galectin-3, galectin-4, linkerless variant of galectin- 4 and both, that is, short and long, versions of galectin-8) in the concentration range of $200-800 \mathrm{ng} / \mathrm{ml}$ over the course of 3 weeks. The experiments revealed that the activity depends on the type of galectin and its concentration (fig. $2 \mathrm{a}-\mathrm{f}$ ). The strongest induction of the conversion of fibroblasts to MFBs was achieved using homodimeric galectin-1 (fig. 2a). Impairing its carbohydrate-binding activity by introducing the E71Q mutation led to a drastic reduction of its stimulatory ability (fig. 2b). Activity was also found for galectin-3, depending on the presence of the N-terminal stalk, because the truncated version of this galectin was inactive (fig. 2c, d). The natural version of tandem-repeat galectin-4 (fig. 2e) was also active concerning MFB formation. Parallel experiments 

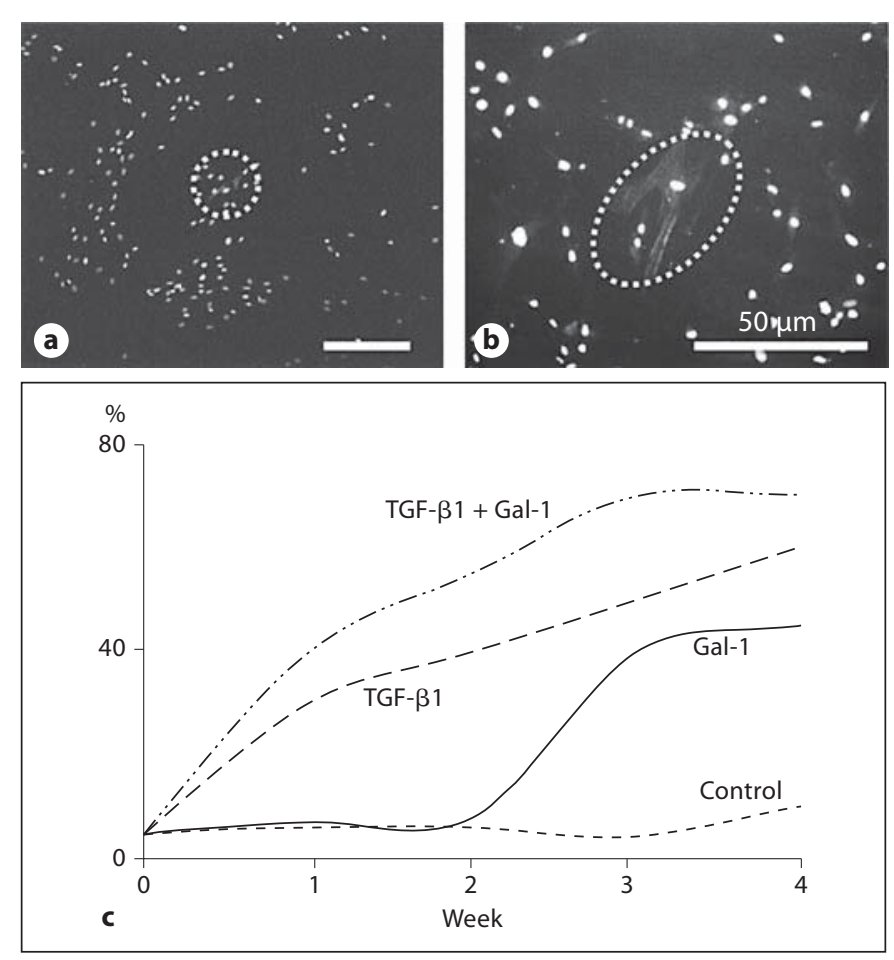

Fig. 1. Detection of smooth muscle actin in control cultures of dermal fibroblasts $(\mathbf{a}, \mathbf{b})$ and illustration of the influence of TGF$\beta 1$ and galectin-1 $(200 \mathrm{ng} / \mathrm{ml})$ on the formation of smooth muscle actin-positive myofibroblasts. The number of myofibroblasts was estimated as percentage of the number of cell nuclei occurring in smooth muscle actin-positive myofibroblast clusters related to the total number of nuclei in the evaluated area in three independent experiments. The representative figures demonstrating at the same level of magnification the control culture (d), the effect of TGF- $\beta 1$ (e), TGF- $\beta 3$ (f), combination of TGF- $\beta 1$ with two concentrations of galectin-1 $(\mathbf{c}, \mathbf{g}, \mathbf{h})$ and with antibody neutralizing galectin-1 are also included (i). The results show that myofibro-
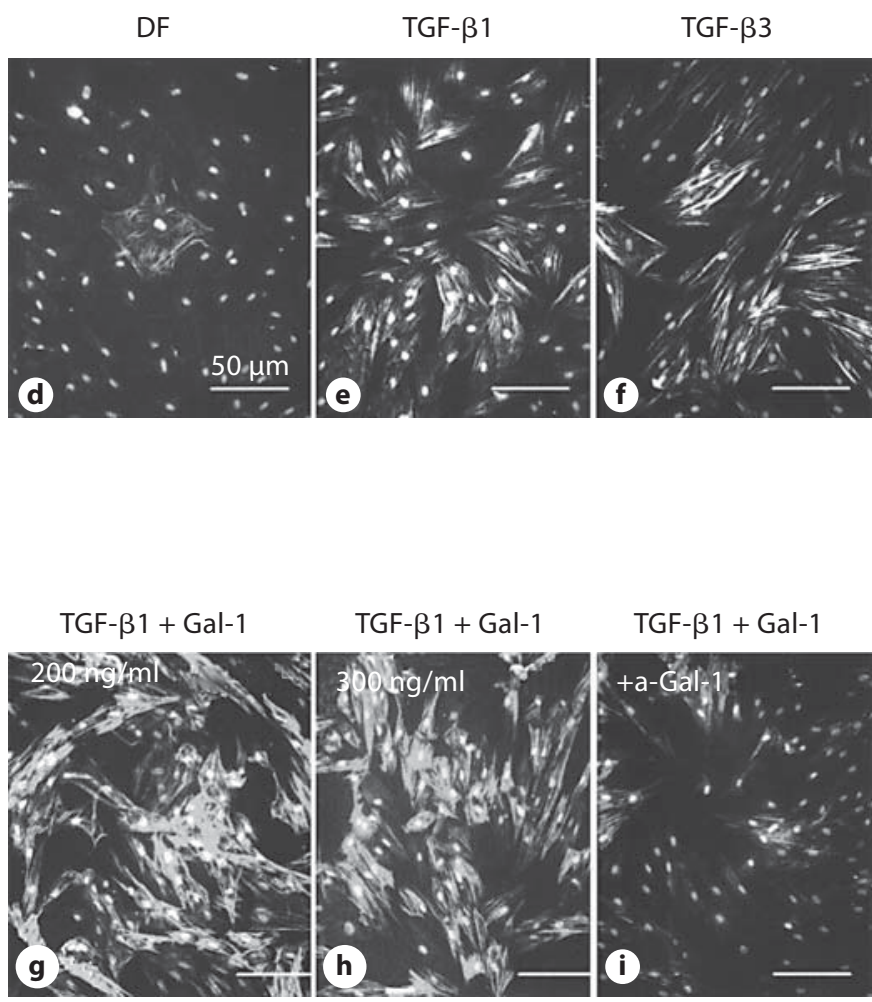

blasts with low extent of smooth muscle actin expression are very rarely present in control (untreated) cultures of dermal fibroblasts (a, b, encircled). While the number of myofibroblasts is very low and stable in control cultures (a-d) during the testing period, TGF- $\beta 1$ (c, e), TGF- $\beta 3$ (f) and galectin-1 (c) have stimulatory effect on myfibroblast formation, with additive effect when both substances were applied simultaneously $(\mathbf{c}, \mathbf{g}, \mathbf{h})$, the TGF being added to the system at the beginning of cell culture. The introduction of anti-galectin-1 antibody to the system, in which both the TGF- $\beta 1$ and galectin- 1 were applied simultaneously, strongly reduced the number of myofibroblasts (i).

concentration of TGF- $\beta 1$ in conditioned media, that is, controls and samples from cultures in the presence of galectin-1, galectin-3 and galectin-7. Except for two values (fig. 3), detectable concentrations of TGF- $\beta 1$ were evidently not reached. Comparing these data with the physiological levels of TGF- $\beta 1$ in serum [Lin et al., 2009] and with the concentration necessary for conversion (10 ng/ $\mathrm{ml})$, the measured data provided no evidence for TGF- $\beta 1$ secretion upon galectin presence (fig. 3). As expected, both TGF- $\beta 1$ and TGF- $\beta 3$ induced dermal fibroblasts to generate MFBs (fig. 1c-f). When galectin-1 was added to medium containing TGF- $\beta 1$, an additive effect was observed (fig. 1c, g, h). An antibody against the lectin strongly reduced the formation of MFBs (fig. 1i). 

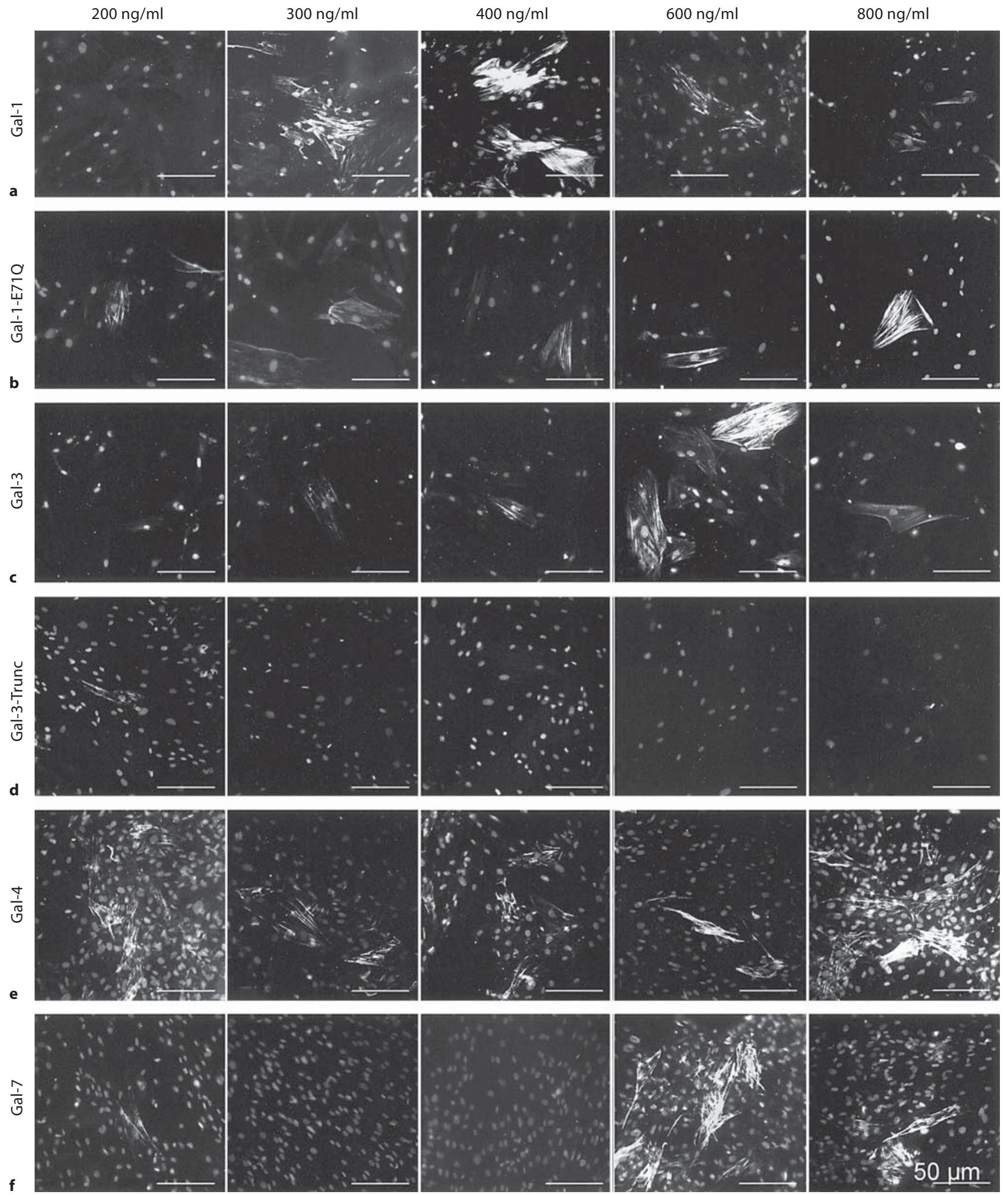

Fig. 2. Influence of galectins (Gal), Gal-1, Gal-1-E71Q mutant, Gal-3, the proteolytically truncated Galectin-3 (Gal-3-Trunc), Gal-4 and Gal-7 on the formation of myfibroblasts detected by monitoring smooth muscle actin. Nuclei are counterstained by DAPI. 
Fig. 3. Influence of wild-type galectin-1 and its E71Q mutant on the concentration of TGF- $\beta 1$ in cultures of normal dermal fibroblasts (DF). Two measured concentrations (arrows) are very low in comparison to the TGF- $\beta 1$ concentration used for stimulating myofibroblast formation $(\mathrm{x})$ and that found in normal plasma in humans (y).

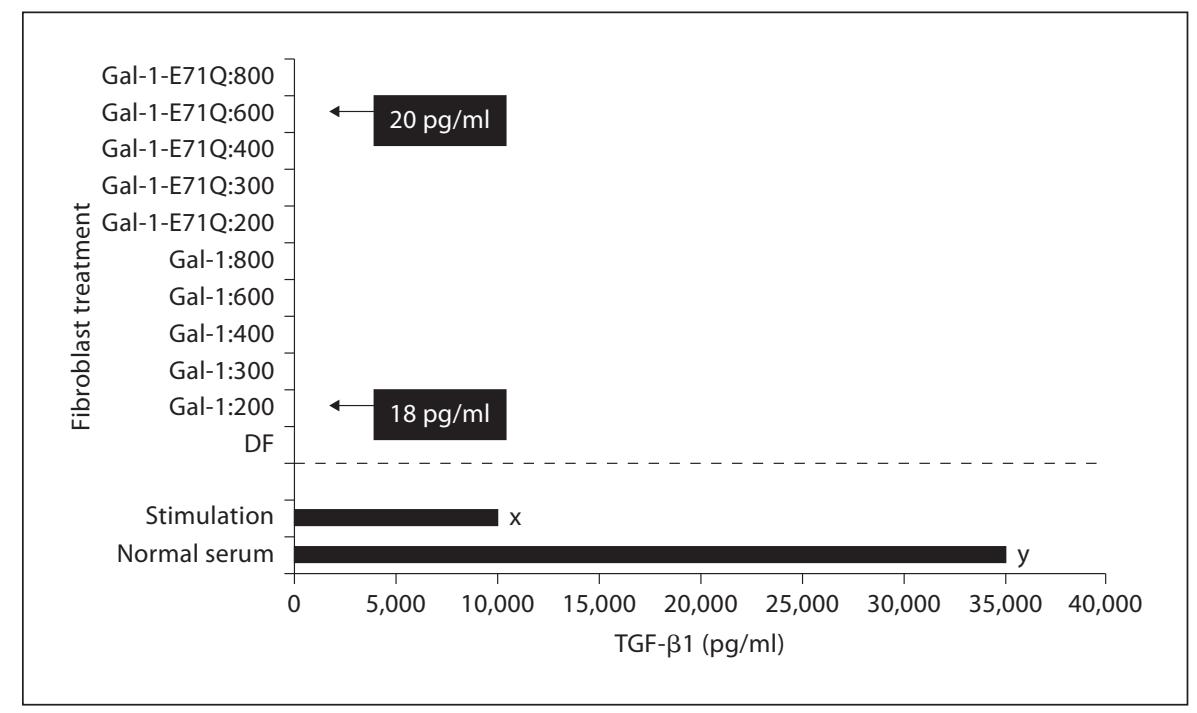

Galectins Stimulate ECM Formation

We found that the presence of certain galectins led to the appearance of three-dimensional ECM scaffold (fig. $4 \mathrm{a}-\mathrm{h}$ ). Namely, galectin-1, galectin-4 and galectin-7 (fig. $4 \mathrm{~b}, \mathrm{c}, \mathrm{d}, \mathrm{g}, \mathrm{h}$ ) were responsible for the marked production of a complex three-dimensional network of fibers of $140 \pm 60 \mathrm{~nm}$ in diameter. The galectin-1-E71Q mutant was not able to stimulate ECM production (fig. $4 \mathrm{~b}-\mathrm{d}$ ). Other galectins, including galectin-3, were inactive (fig. 4e, f). ECM fibers were composed from fibronectin (fig. 4a-h, 5a, b) and galectin-1 (fig. 5b, e) with colocalization of these two proteins. When probing for collagen-1 and collagen-3, only a very limited amount of collagen-1 was detected in cultures of the stimulated cells (fig. $5 c$, d). Interestingly, using galectin- 4 and galectin- 7 as inductors, the cultured cells were able to produce an ECM enriched in galectin-1 but not in galectin- 4 or galectin-7 (fig. $5 e, f$ ).

ECM Scaffolds Produced by Galectin-Stimulated

Fibroblasts Influence Phenotype of Cultured

\section{Keratinocytes}

To determine whether such an ECM scaffold produced by fibroblasts/MFBs can find applications in cell culture, we next tested the panel of galectins given in the introduction in culture of keratinocytes without feeder cells. Keratinocytes cultured on these coverslips with a three-dimensional ECM network composed from fibronectin and galectin-1 appeared to resorb this matrix (fig. 5g). In comparison to control experiments (fig. 5h, i), the cells' size was significantly smaller, and they expressed keratin-19 as one of the putative markers of poor- ly differentiated keratinocytes. When the coverslips with the substratum were pretreated with glutaraldehyde to crosslink its components, the ECM lattices became resistant to resorption, but the cells remained negative for keratin-19 (not shown).

\section{In vivo Experiments}

Galectins Influence Wound Healing

To take the observed in vitro activity to the in vivo level, we finally addressed the issue of rat skin-wound healing in the presence of galectins (galectin-1, galectin1-E71Q mutant, galectin-3). No remarkable effect on wound re-epithelialization was observed when the animals were treated with any of the tested galectins as the extent of re-epithelialization was identical with that seen with galectin-free controls and the wounds were completely re-epithelialized 3 weeks after the surgery (fig. $6 \mathrm{a}-\mathrm{d})$. Measuring the extent of wound contraction also revealed no effect of galectins at day 7, when wound reepithelialization was only partial. Continued observation revealed an effect for galectin-1. Compared to its control (E71Q), galectin-1 gave a positive result after 21 days, not seen for galectin-3 (fig. 6e-i).

\section{Discussion}

Our study addresses four issues pertinent to evaluating the potential of galectins for application in cell-based and topical therapy modalities in a long-term perspective. Building on histopathological observations of the 

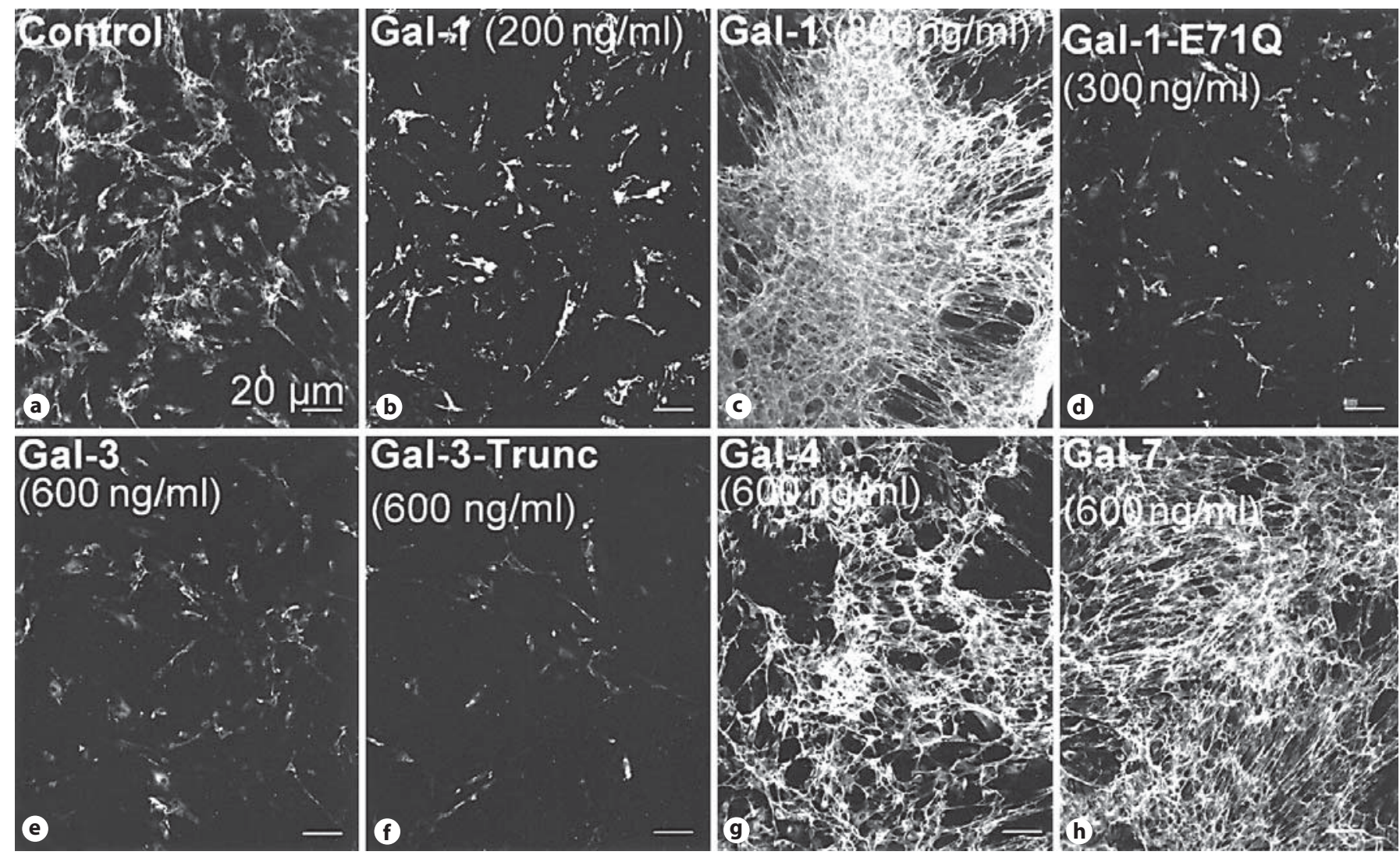

Fig. 4. Immunocytochemical detection of fibronectin in control culture of dermal fibroblasts (a) and fibroblasts treated with galectin-1 in two concentrations (b, c), with mutated galectin-1 (d), galectin-3 (e), truncated version of galectin-3 (f), galectin-4 (g) and galectin-7 (h) simultaneously. The extensive production of a three-dimensional fibronectin network was observed in cultures treated with galectin-1 $(300 \mathrm{ng} / \mathrm{ml}, \mathbf{c})$, galectin-4 (g) or galectin-7 (h).

presence of stromal galectin and the capacity of galectin-1 to direct mesenchymal stem cells and fetal fibroblasts towards the myogenic lineage as described in the introduction, we tested this protein on adult dermal fibroblasts and found that it was able to convert them to MFBs at submicrogram/milliliter concentrations. Of note, this activity was abolished when the lectin site's functionality was impaired by the E71Q mutation. The previously reported TGF-like activity of rat galectin-1 on DNA synthesis and anchorage-independent growth of 3T3 cells, in contrast, was not sensitive to haptenic sugar inhibition [Yamaoka et al., 1993]. The induction of TGF$\beta$ secretion as a downstream effector could be excluded, while an additive effect of galectin-1 and the growth factor could be documented.

This ability of galectin-1 led us to monitor other human galectins, that is, to explore the functionality profile among these homologous proteins. We detected a conspicuous functional divergence within the class of prototype and tandem-repeat-type galectins. Also, a requirement for the presence of the stalk region in galectin-3, which is responsible for cross-linking activity, was seen. Evidently, even structurally closely related family members can differ markedly in terms of functionality. Such divergence has previously been seen for the growth regulation of human neuroblastoma and pancreatic carcinoma cells as well as activated T cells [Kopitz et al., 2001, 2010; Sturm et al., 2004; Sanchez-Ruderisch et al., 2010]. Since galectin secretion can make these proteins available in the stroma, this described ability to increase the presence of MFBs may eventually contribute to tumor progression, analogously to TGF- $\beta$, for example in colon cancer where galectin-1 and galectin- 4 in combination have a strongly negative impact on prognosis [Nagy et al., 

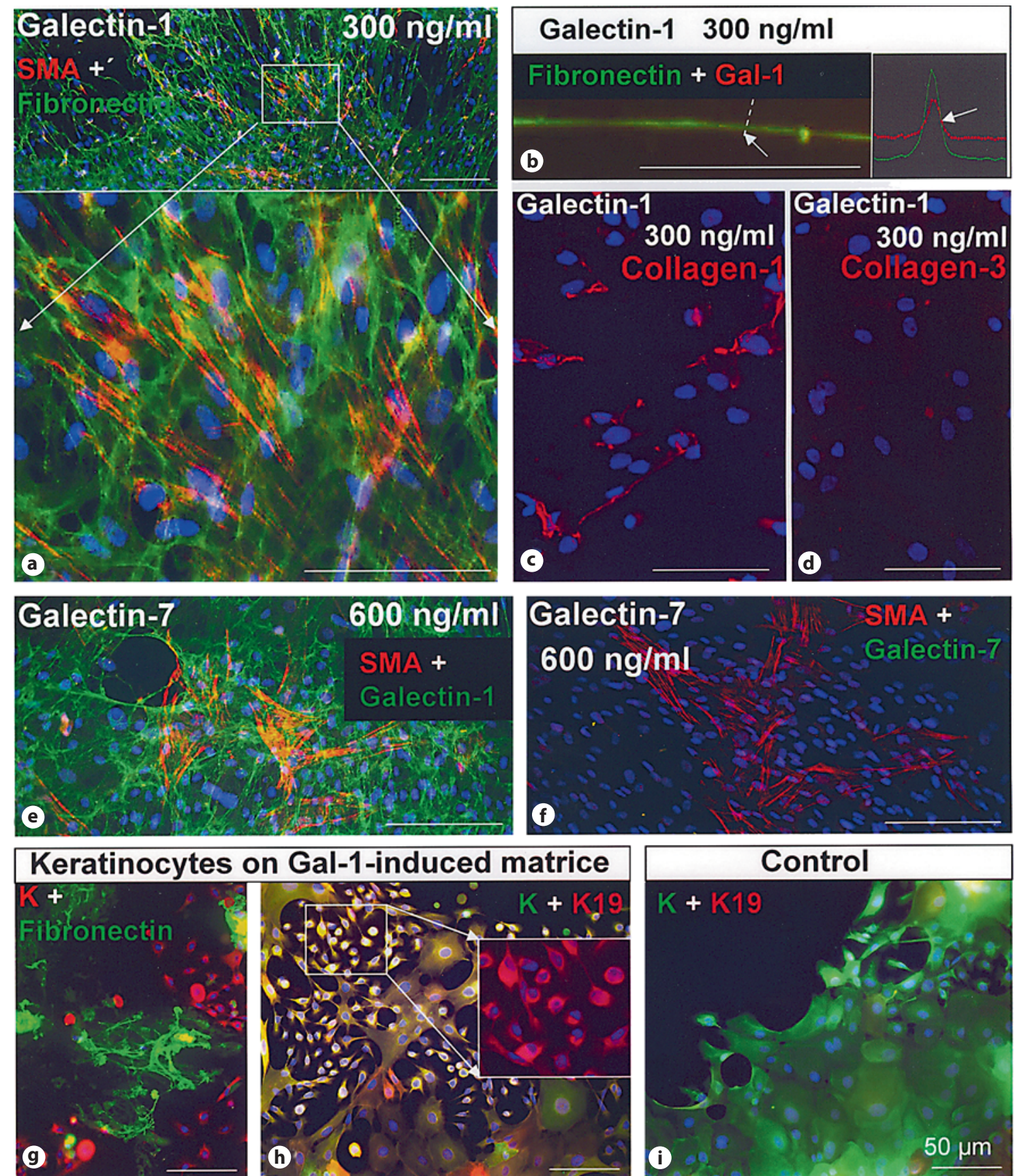

Fig. 5. Influence of galectin-1 on the production of fibronectin (green signal, a, b) and a galectin-1 (red signal, b)-containing three-dimensional network, compared to a weak effect on collagen-1 synthesis (red signal, c) and a lack of effect on collagen-3 synthesis (red signal, d). Analyzing the presence of fibronectin and galectin-1 (b), both proteins were detected and the distribution measured by fluorescence profiles. Other types of galectins such as galectin-7 also induced the production of galectin-1 (green signal, e) but not of galectin-7 (green signal, f). Visualiza- tion of smooth muscle actin (red signal, a e, e, f) indicated that myofibroblasts are located in the extracellular matrix network. When keratinocytes (red signal-keratin, $\mathbf{g}$ ) were seeded on an ECM lattice formed by galectin-1-induced fibroblasts/myofibroblasts, resorption of ECM lattice was apparent (green signal, fibronectin, g). These keratinocytes were very small and expressed keratin-19 (red signal, h) in addition to pan-kreatin (green, h). Keratinocytes cultured on coverslips are shown for comparison (i). Nuclei are counterstained by DAPI. 


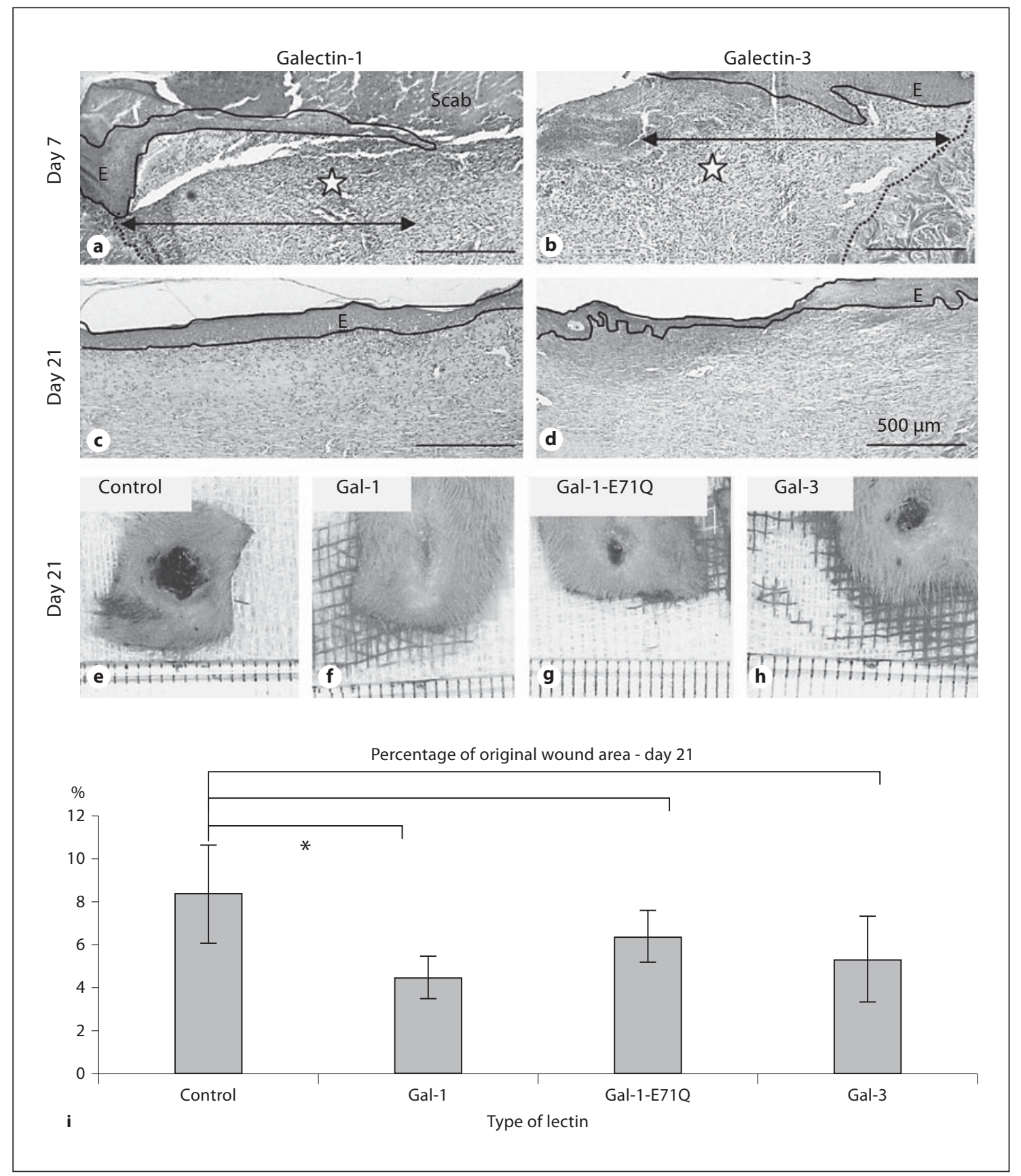

Fig. 6. Effect of galectins on the re-epithelialization and contraction of skin wounds. Partial re-epithelialization as reached one week after the administration of galectin-1 (a) or galectin-3 (b). The double-headed arrow marks the distance between the wound margin (dotted line) and the edge of regenerating epithelium. The complete reaction is shown 3 weeks after the surgery $(\mathbf{c}, \mathbf{d})$. Galectins $(\mathbf{e}-\mathbf{i})$ stimulated wound contraction in comparison to physiological saline as a control $(\mathbf{e}, \mathbf{i})$ with the highest efficiency seen for wild-type galectin-1 (f, i), which surpassed the threshold for statistical significance after 3 weeks $(p<0.05)$. 
2003] or in breast cancer, where stromal galectin-3 is a negative prognostic factor [Moisa et al., 2007]. This study thus broadens the experimental evidence for galectins as effectors for MFB generation and delineates marked activity differences.

In addition to cellular conversion, the formation of a complex scaffold rich in fibronectin and galectin-1 by MFBs was detected immunocytochemically. Its assumed bioactivity was ascertained by culturing human keratinocytes on this substratum without feeder cells. Strikingly, their phenotype, with a small diameter and keratin-19 expression, is evocative of a low differentiation status [Dvořánková et al., 2005], implying a beneficial role also in wound healing in vivo together with MFB generation [Kwon et al., 2006]. In the last part of our study, we report a positive effect of galectin-1 application on skin wound healing in rats at day 21 after surgery.

In summary, this study describes a TGF- $\beta$-like activity of galectin-1 on MFB generation from adult dermal fibroblasts and detects functional divergence in this re- spect among this family of human lectins. The matrix scaffold produced in vitro supports keratinocyte culture without feeder cells. In vivo, the application of galectin-1 appears favorable for skin-wound healing after 3 weeks. Beyond wound healing, the described addition to the activity profile of galectins, considered as biomaterials, can also have relevance for explaining the physiological significance of the presence of stromal galectin in tumors.

\section{Acknowledgements}

This study was supported by the Ministry of Education, Youth and Sports of the Czech Republic projects No. MSM 0021620806 and $1 \mathrm{M} 0538$, the Charles University in Prague, the EC research program GlycoHIT (contract ID 260600) and the Verein zur Förderung des biologisch-technologischen Fortschritts in der Medizin e.V. (Heidelberg, Germany). The authors are grateful to Dr. James Dutt for the language revision and to Iva Burdová, Dr. Tomáš Vasilenko and Magdaléna Majnušová for excellent technical assistance.

\section{References}

André, S., S. Kojima, N. Yamazaki, C. Fink, H. Kaltner, K. Kayser, H.-J. Gabius (1999) Galectins -1 and -3 and their ligands in tumor biology. J Cancer Res Clin Oncol 125: 461474.

André, S., Z. Pei, H.-C. Siebert, O. Ramström, H.-J. Gabius (2006) Glycosyldisulfides from dynamic combinatorial libraries as O-glycoside mimetics for plant and mammalian lectins: their reactivities in solid-phase and cell assays and conformational analysis by molecular dynamics simulations. Bioorg Med Chem 14: 6314-6323.

André, S., H. Sanchez-Ruderisch, H. Nakagawa, M. Buchholz, J. Kopitz, P. Forberich, W. Kemmner, C. Böck, K. Deguchi, K.M. Detjen, B. Wiedenmann, M. von Knebel Doeberitz, T.M. Gress, S.-I. Nishimura, S. Rosewicz, H.-J. Gabius (2007) Tumor suppressor p16 ${ }^{\text {INK4a }}$ : modulator of glycomic profile and galectin-1 expression to increase susceptibility to carbohydrate-dependent induction of anoikis in pancreatic carcinoma cells. FEBS I 274: 3233-3256.

André, S., F. Sansone, H. Kaltner, A. Casnati, J. Kopitz, H.-J. Gabius, R. Ungaro (2008) Calix[n]arene-based glycoclusters: bioactivity of thiourea-linked galactose/lactose moieties as inhibitors of binding of medically relevant lectins to a glycoprotein and cellsurface glycoconjugates and selectivity among human adhesion/growth-regulatory galectins. ChemBioChem 9: 1649-1661.
Beer, A., S. André, H. Kaltner, M. Lensch, S. Franz, K. Sarter, C. Schulze, U.S. Gaipl, P. Kern, M. Herrmann, H.-J. Gabius (2008) Human galectins as sensors for apoptosis/necrosis-associated surface changes of granulocytes and lymphocytes. Cytometry 73A: 139-147.

Brenmoehl, J., S.N. Miller, C. Hofmann, D. Vogl, W. Falk, J. Schölmerich, G. Rogler (2009) Transforming growth factor- $\beta 1$ induces intestinal myofibroblast differentiation and modulates their migration. World J Gastroenterol 15: 1431-1442.

Čada, Z., K. Smetana Jr, L. Lacina, Z. Plzáková, J. Štork, H. Kaltner, R. Russwurm, M. Lensch, S. André, H.-J. Gabius (2009) Immunohistochemical fingerprinting of the network of seven adhesion/growth-regulatory lectins in human skin and detection of distinct tumorassociated alterations. Folia Biol (Praha) 55: 145-152.

Chan, J., K. O'Donoghue, M. Gavina, Y. Torrente, N. Kennea, H. Mehmet, H. Stewart, D.J. Watt, J.E. Morgan, N.M. Fisk (2006) Galectin-1 induces skeletal muscle differentiation in human fetal mesenchymal stem cells and increases muscle regeneration. Stem Cells 24: 1879-1891.

Chang, Y.-Y., S.-J. Cheb, H.-C. Liang, H.-W. Sung, C.-C. Lin, R.-R. Juany (2004) The effect of galectin-1 on 3T3 cell proliferation on chitosan membrane. Biomaterials 25: 36033611.
De Wever, O., P. Demetter, M. Mareel, M. Bradle (2008) Stromal myofibroblasts are drivers of invasive cancer growth. Int J Cancer 123: 2229-2238.

Dvořánková, B., K. Smetana Jr., M. Chovanec, L. Lacina, J. Štork, Z. Plzáková, M. Galovičová, H.-J. Gabius (2005) Transient expression of keratin K19 is induced in originally negative interfollicular epidermal cells by adhesion of suspended cells. Int J Mol Med 16: 525-531.

Fischer, C., H. Sanchez-Ruderisch, M. Welzel, B Wiedenmann, T. Sakai, S. André, H.-J. Gabius, L. Khachigian, K.M. Detjen, S. Rosewicz (2005) Galectin-1 interacts with the $\alpha_{5} \beta_{1}$ fibronectin receptor to restrict carcinoma cell growth via induction of p21 and p27. J Biol Chem 280: 37266-37277.

Gabius, H.-J. (ed) (2009) The Sugar Code. Fundamentals of Glycosciences. Weinheim, Wiley-VCH.

Gabius, H.-J., R. Engelhardt, F. Cramer, R. Bätge, G.A. Nagel (1985) Pattern of endogenous lectins in a human epithelial tumor. Cancer Res 45: 253-257.

Goldring, K., G.E. Jones, R. Thiagarajah, D.J. Watt (2002a) The effect of galectin-1 on the differentiation of fibroblasts and myoblasts in vitro. J Cell Sci 115: 355-366.

Goldring, K., G.E. Jones, C.A. Sewry, D.J. Watt (2002b) The muscle-specific marker desmin is expressed in a proportion of human dermal fibroblasts after their exposure to galectin-1. Neuromuscul Disord 12: 183-186. 
Hennet, T. (2009) Diseases of glycosylation; in Gabius, H.-J. (ed): The Sugar Code. Fundamentals of Glycosciences. Weinheim, WileyVCH, pp. 365-383.

Hinz, B. (2007) Formation and function of the myofibroblast during tissue repair. J Invest Dermatol 127: 526-537.

Honke, K., N. Taniguchi (2009) Animal models to delineate glycan functionality; in Gabius, H.-J. (ed): The Sugar Code. Fundamentals of Glycosciences. Weinheim, Wiley-VCH, pp 385-401.

Kadri, T., J.-J. Lataillade, C. Doucet, A. Marie, I. Ernou, P. Bourin, R. Joubert-Caron, M. Caron, D. Lutomski (2005) Proteomic study of galectin-1 expression in human mesenchymal stem cells. Stem Cells Dev 14: 204-212.

Kaltner, H., K. Seyrek, A. Heck, F. Sinowatz, H.J. Gabius (2002) Galectin-1 and galectin-3 in fetal development of bovine respiratory and digestive tracts. Comparison of cell typespecific expression profiles and subcellular localization. Cell Tissue Res 307: 35-46.

Klíma, J., L. Lacina, B. Dvořánková, D. Herrmann, J.W. Carnwath, H. Niemann, H. Kaltner, S. André, J. Motlík, H.-J. Gabius, K. Smetana Jr (2009) Differential regulation of galectin expression/reactivity during wound healing in porcine skin and in cultures of epidermal cells with functional impact on migration. Physiol Res 58: 873-884.

Kopitz, J., C. von Reitzenstein, S. André, H. Kaltner, J. Uhl, V. Ehemann, M. Cantz, H.-J. Gabius (2001) Negative regulation of neuroblastoma cell growth by carbohydrate-dependent surface binding of galectin-1 and functional divergence from galectin-3. J Biol Chem 276: 35917-3523.

Kopitz, J., M. Bergmann, H.-J. Gabius (2010) How adhesion/growth-regulatory galectins- 1 and -3 attain cell specificity: case study defining their target on neuroblastoma cells (SK-N-MC) and marked affinity regulation by affecting microdomain organization of the membrane. IUBMB Life 62 : 624-628.

Kübler, D., C.W. Hung, T.K. Dam, J. Kopitz, S. André, H. Kaltner, M. Lohr, J.C. Manning, L. He, H. Wang, A. Middelberg, C.F. Brewer, J. Reed, W.D. Lehmann, H.-J. Gabius (2008) Phosphorylated human galectin-3: facile largescale preparation of active lectin and detection of structural changes by CD spectroscopy. Biochim Biophys Acta 1780: 716-722.
Kwon, Y.-B., H.-W. Kim, D.-H. Roh, S.-Y. Yoon, R.-M. Baek, J.-Y. Kim, H. Kweon, K.-G. Lee, Y.-H. Park, J.-H. Lee (2006) Topical application of epidermal growth factor accelerates wound healing by myofibroblast proliferation and collagen synthesis in rat. J Vet Sci 7: 105-109.

Labský, J., B. Dvoránková, K. Smetana Jr., Z. Holíková, L. Brož, H.-J. Gabius (2003) Mannosides as crucial part of bioactive supports for cultivation of human epidermal keratinocytes without feeder cells. Biomaterials 24: 863-872.

Lacina, L., K. Smetana Jr., B. Dvořánková, R. Pytlík, L. Kideryová, L. Kučerová, Z. Plzáková, J. Štork, H.-J. Gabius, S. André (2007) Stromal fibroblasts from basal cell carcinoma affect phenotype of normal keratinocytes. Brit J Dermatol 156: 819-829.

Lin, Y., K. Nakachi, Y. Ito, S. Kikuchi, A. Tamakoshi, K. Yagyu, Y. Watanabe, Y. Inaba, K. Tajima (2009) Variations in serum transforming growth factor- $\beta 1$ levels with gender, age and lifestyle factors of healthy Japanese adults. Dis Markers 27: 23-28.

Moisa, A., P. Fritz, A. Eck, H.-D. Wehner, T. Mürdter, W. Simon, H.-J. Gabius, (2007) Growth/adhesion-reglatory tissue lectin galectin-3: stromal presence but not cytoplasmic/nuclear expression in tumor cells as a negative prognostic factor in breast cancer. Anticancer Res 27: 2131-2140.

Nagy, N., H. Legendre, O. Engels, S. André, H. Kaltner, K. Wasano, Y. Zick, J.C. Pector, C. Decaestecker, H.-J. Gabius, I. Salmon, R. Kiss (2003) Refined prognostic evaluation in colon cancer using immunohistochemical galectin fingerprinting. Cancer 97: 1849-1858.

Okano, K., K. Uchida, K. Nitta, T. Hayashida (2008) Galectin-1 suppresses $\alpha 2$ (I) collagen through Smad3 in renal epithelial cells. Cell Mol Life Sci 65: 3304-3311.

Saal, I., N. Nagy, M. Lensch, M. Lohr, J.C. Manning, C. Decaestecker, S. André, R. Kiss, I. Salmon, H.-J. Gabius (2005) Human galectin-2:expression profiling by RT-PCR/immunohistochemistry and its introduction as histochemical tool for ligand localization. Histol Histopathol 20: 1191-1208.

Sanchez-Ruderisch H., C. Fischer, K. M. Detjen, M. Welzel, A. Wimmel, J. C. Manning, S. André, H.-J. Gabius (2010) Tumor suppressor $\mathrm{p} 16^{\text {INK4a }}$ : downregulation of galectin-3, an endogenous competitor of the pro-anoikis effector galectin-1, in a pancreatic carcinoma model. FEBS J 277: 3552-3563.
Sarter, K., S. André, H. Kaltner, M. Lensch, C. Schulze, V. Urbonaviciute, G. Schett, M. Herrmann, H.-J. Gabius (2009) Detection and chromatographic removal of lipopolysaccharide in preparations of multifunctional galectins. Biochem Biophys Res Commun 379: 155-159.

Strnad, H., L. Lacina, M. Kolář, Z. Čada, Č. Vlček, B. Dvořánková, J. Betka, J. Plzák, M. Chovanec, J. Šáchová, J. Valach, M. Urbanová, K. Smetana Jr. (2010) Head and neck squamous cancer stromal fibroblasts produce growth factors influencing phenotype of normal human keratinocytes. Histochem Cell Biol 133: 201-211.

Sturm, A., M. Lensch, S. André, H. Kaltner, B. Wiedenmann, S. Rosewicz, A.U. Dignass, H.-J. Gabius (2004) Human galectin-2: novel inducer of $\mathrm{T}$ cell apoptosis with distinct profile of caspase activation. J Immunol 173: 3825-3837.

Szabo, P., T.K. Dam, K. Smetana Jr, B. Dvořánková, D. Kübler, C.F. Brewer, H.-J. Gabius (2009) Phosphorylated human lectin galectin-3: analysis of ligand binding by histochemical monitoring of normal/malignant squamous epithelia and by isothermal titration calorimetry. Anat Histol Embryol 38: 68-75.

Villalobo, A., A. Nogales-Gonzalés, H.-J. Gabius (2006) A guide to signaling pathways connecting protein-glycan interaction with the emerging versatile effector functionality of mammalian lectins. Trends Glycosci Glycotechnol 18: 1-37.

Voog, J., D.L. Jones (2010) Stem cells and the niche: a dynamic duo. Cell Stem Cell 6: 103115.

Wang, J., Z.-H. Lu, H.-J. Gabius, C. RohowskyKochan, R.W. Ledeen, G. Wu (2009) Crosslinking of GM1 ganglioside by galectin-1 mediates regulatory $\mathrm{T}$ cell activity involving TRPC5 channel activation: possible role in suppressing experimental autoimmune encephalomyelitis. J Immunol 182: 4036-4045.

Yamaoka, K., S. Ohno, H. Kawasaki, K. Suzuki (1993) Mammalian lectin as transforming growth factor; in Gabius, H.-J., S. Gabius (eds): Lectins and Glycobiology. Heidelberg, Springer Verlag, pp. 492-499. 